\title{
Truncated Precursor of Feline calicivirus Major Capsid Protein: A Product Relevant for Replication, or an Aberrant Translation Artifact?
}

\author{
Ángel L. Álvarez Francisco Parra \\ Departamento de Bioquímica y Biología Molecular, Instituto Universitario de Biotecnología de Asturias (IUBA), \\ Universidad de Oviedo, Oviedo, Spain
}

In a recent JVI paper by Urban and Luttermann [1], a truncated version of VP1 precursor (tLC-VP1) synthesized directly from the genomic RNA (gRNA) was detected for the first time for Feline calicivirus (FCV). Through a series of comprehensive reverse genetics experiments using $3 \mathrm{D}^{\mathrm{Pol}} / \mathrm{LC}$-cleavage mutants, RIPA, luciferase reporter assays and deletion mutants, the authors demonstrated translational activity leading to tLC-VP1 synthesis starting at AUG codon 86, which they claim to be the second start codon within the whole LC-VP1 sequence. The authors suggested that a novel, scanning-independent, rather unknown translation initiation mechanism may be responsible for tLC-VP1 synthesis and identified specific sequences upstream of M86 that appear to be important for its efficiency. For example, in a reporter assay, the S3S mutant, lacking a stem-loop naturally occurring close to the LC-VP1 first AUG (M1), showed a dramatically decreased luciferase expression due to impaired translation initiation at M86. Furthermore, in a multiple-step growth assay, the S3S mutant showed decreased titers at early points of the curve, compared to wild-type FCV, though all assayed viruses reached similar end point titers. Based on these findings, the authors

karger@karger.com www.karger.com/int

Karger:

GOPEN ACCESS
C 2021 The Author(s).

Published by S. Karger AG, Basel

This is an Open Access article licensed under the Creative Commons Attribution-NonCommercial-4.0 International License (CC BY-NC) (http://www.karger.com/Services/OpenAccessLicense), applicable to the online version of the article only. Usage and distribution for commercial purposes requires written permission. speculate that tLC-VP1 has a role during early phases of virus replication and claim that "all caliciviruses express VP1 from the gRNA" because it is essential and required early upon infection. We expected that the authors discussed the well-established fact that all caliciviruses encapsidate the subgenomic RNA (sgRNA) within the virions $[2,3]$, that is readily translated upon infection and the biological significance of producing a rather redundant tLC-VP1 in this context.

The authors based their work on the sequence of FCV 2024 vaccine strain (GenBank AF479590.1) that contain no additional methionine residue between M1 and M86. However, a close look at other GenBank FCV sequences reveals that most FCV strains do possess another inframe AUG start codon within the LC-VP1 sequence at position 38 (M38), which has been overlooked in this work (Fig. 1a). In addition, when we compared the LCVP1 sequence from FCV strain Urbana with those of several members of the Vesivirus genus we found no inframe AUG start codon between the first ORF2 AUG (M1) and the putative proteolytic cleavage site responsible for LC excision and release of mature VP1, except for Allston calicivirus (M122, M134, M141) and SMSV-8 


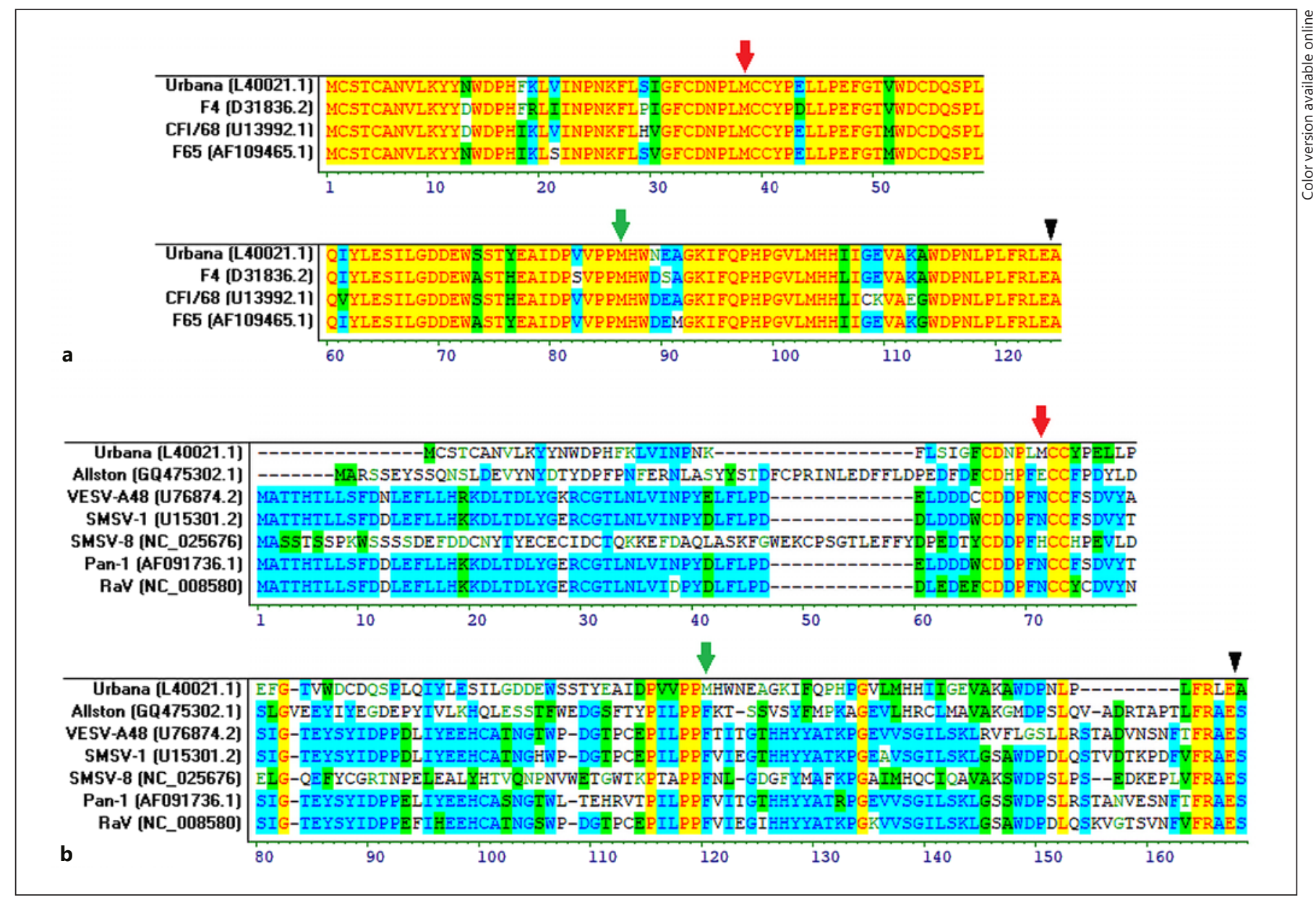

Fig. 1. Multiple alignment of the amino acid sequences of major capsid protein precursor (LC-VP1) from Feline calivirus (FCV) strains Urbana, F4, CFI/68, and F65 (a) and from the vesiviruses FCV strain Urbana, Allston calicivirus (b), Vesicular exanthema of swine virus strain A48 (VESV-A48), San Miguel sea lion virus serotype 1 (SMSV-1), San Miguel sea lion virus serotype 8 (SMSV-8), Primate calicivirus Pan-1 (Pan-1), and Rabbit vesivirus (RaV). GenBank accession numbers are shown in parenthesis. The third methionine in LC-VP1 ORF (M86), referred to as the second one

(M127, M135) that encode methionine residues far downstream of M86 (Fig. 1b).

We were also concerned about the conclusion of tLC-VP1 being essential for virus replication, which we found very preliminary. Considering the extremely low MOI used (0.0003), pipetting variations or very small differences in the number of cells per culture vessel may have a great impact on titers at early time points, especially when TCID50 method is used instead of plaque assay. by Urban and Luttermann, is indicated with a green arrow. The actual second methionine within LC-VP1 ORF (M38) is indicated with a red arrow. The LC/VP1 proteolytic cleavage site (E124/ A125) is indicated with a black arrowhead. Amino acid similarity color legend: yellow background = identical, cyan background = conservative, green background = block of similar, green font/ white background = weakly similar, and black font/white background $=$ non-similar.

That being noticed, we would like to thank the authors for their contribution and kindly invite them to debate on the following questions: Why a tLC-VP1 would be synthesized from the gRNA if the full VP1 can be produced from the encapsidated sgRNA immediately after infection? Could tLC-VP1 be a minor translation product exclusive of the FCV strain used? Could a larger tLC-VP1 version be also expressed in other FCV strains, starting at M38? Why a gRNA-derived product supposedly relevant for calicivirus replication initiation would be absent in most vesiviruses (except FCV)? 


\section{Conflict of Interest Statement}

The authors declare no conflict of interest.

\section{Author Contributions}

A.L.A. conceptualized the work and wrote the manuscript. F.P. conceptualized the work, provided supervision, and revised the manuscript.

\section{References}

1 Urban C, Luttermann C. Major capsid protein synthesis from the genomic RNA of feline calicivirus. J Virol. 2020;94(15).

2 Meyers G, Wirblich C, Thiel HJ. Genomic and subgenomic RNAs of rabbit hemorrhagic disease virus are both protein-linked and packaged into particles. Virology. 1991; 184(2):677-86

3 Neill JD. The subgenomic RNA of feline calicivirus is packaged into viral particles during infection. Virus Res. 2002;87(1):8993. 